\title{
Initial Stability of Type-2 Tibial Defect Treatments
}

\author{
B Frehill ${ }^{1}$, A Crocombe ${ }^{1}$, Cirovic $^{1}$, Y Agarwal $^{1}$ and N Bradley $^{2}$ \\ ${ }^{1}$ Mechanical, Medical and Aerospace Engineering, University of Surrey, UK \\ ${ }^{2}$ Royal Surrey County Hospital, Guildford, UK
}

Abstract: Treatment of proximal tibial defects is important to the survival of tibial prosthesis after total knee replacement. The objective of this finite element study was to determine a better understanding of the stresses produced by different treatment options for moderate uncontained type- 2 defects. Methods analysed were the use of metal wedges, metal blocks, cement wedges and cement blocks for two defect angles of $15^{\circ}$ and $30^{\circ}$. The effect of a stem extension on the stress profiles was also analysed for each defect treatment and angle to establish the necessity of these extensions and consequent bone removal on the stability of the augments. Equivalent stresses in two regions of interest (ROIs) adjacent to the augments and shear stresses along the bonecement interface of the defect were investigated. The lowest equivalent stresses were found in the metal block augment for both defect angles and ROIs. The highest equivalent stress in the ROIs and shear stress values along the bone-cement interface of the defect were found in the cement wedge augment model for both defect angles. Stem extensions were shown to increase equivalent stresses in the bone closer to the tibial stem but decrease equivalent stresses closer to the cortical bone. The use of a stem extension significantly increased the shear stresses in the cement in all cases except in the metal block model. It is recommended that metal block augments are used without a stem extension in small defect (i.e. peripheral defect angle of $15^{\circ}$ ) TKR procedures.

Keywords: total knee replacement, augment, stem extension, initial stability 


\section{INTRODUCTION}

Tibial defects in the proximal tibia are a common occurrence in patients undergoing primary and revision total knee replacement (TKR). Tibial defects can be central or peripheral, but are more commonly classified according to the Anderson Orthopedic Research Institute (AORI) [1]. In order to maintain tibial component fixation it is important to take corrective measures to ensure a stable platform for the implanted prosthesis. Methods to deal with moderate uncontained AORI type-2 (i.e. defects up to $2 \mathrm{~cm}$ in depth and minor damage to metaphysis) tibial defects include the use of bone cement to fill the defect, resection of the proximal tibia to the level of the defect, the use of bone autograft or allograft to fill the defect, the use of metallic wedge, block and conical augments and the use of a custom prosthesis. The size and depth of the tibial defect is the main factor for the surgeon to consider when choosing the most appropriate method [2]. Resection of bone to the level of the defect is confined to the treatment of shallow defects. Cancellous bone becomes relatively weaker as the level of resection depth increases thus placing the tibial component on weak cancellous bone [3]. The use of bone autograft/allograft results in an increased surgery time thus increasing the risk of infection, the possibility of non-union and collapse/resorption of the graft. The use of allograft also poses a risk of disease transmission [4].

Fracture or loosening of the cement augment in cases where type- 2 defects are filled by cement alone may occur with resulting displacement and loosening compromising the stability of the tibial tray. The use of metal wedge augments has been shown to reduce the deflection of the tray when compared with the use of cement wedges [5] and has a lower failure rate in in-vitro studies [6] and clinical trials [7]. However the use of wedge shaped augments may lead to stress concentrations in the cancellous bone at the inner margin of the tray and augment and an increase in 
shear forces acting on the defect area due to its tapered nature. The use of block augmentation (i.e. a stepped resection filled with cement or a metal block) may eliminate these complications, but would result in resection of valuable bone stock. Previous in-vitro studies [8] have shown no significant difference in stiffness values when stepped shaped defects were filled with a metal compared with a cement augment. Metal conical augments are a new treatment option for more severe type-2 defects which provide extended mechanical support for tibial TKR components [9]. Short-term clinical studies for conical augments have shown strong potential for the use of these devices with effective support for tibial prosthetic components reported in cases of treatment of more severe type-2 defects [10]. However no long-term results are currently available.

The use of stem extensions in conjunction with augments in revision surgery is the usual approach in dealing with proximal tibial defects. This approach may be considered over-conservative and may also result in large difficulties due to internal bone damage should further revision be necessary. The function of stem extensions is usually to prevent rocking and to bypass bone defects or offload stresses from the proximal region to the distal region thus reducing the possibility of loosening in the weaker proximal cancellous bone [9]. The use of augments (wedge and block) to treat tibial defects may eliminate the need for stem extensions and thus preserve native distal bone.

The objective of this study was to determine if the different augmentation procedures have a significant effect on stress patterns in the proximal tibia and to predict the likelihood of failure due to stress concentrations using $2 \mathrm{D}$ finite element (FE) modelling. This is a pre-cursor to a more extensive 3D study. Type-2 defect angles of $15^{\circ}$ and $30^{\circ}$ were examined and treated with wedge (metal and cement) 
augments, block (metal and cement) augments of both sizes and with appropriate depth and bone resection. These models were then extended with a stem extension of $30 \mathrm{~mm}$ to examine if these have any effect on resultant stresses. Equivalent stresses in the bone and shear stresses in the cement can be correlated with failure of the TKR to verify the influence of these augmentation techniques.

\section{MATERIALS AND METHODS}

Two-dimensional, static, FE models were created of a TKR proximal tibia with type-2 defects in the sagittal plane. Peripheral defect angles of $15^{\circ}$ and $30^{\circ}$ were considered while four methods of treating these defects were also examined. The four methods involved filling the defect with a cement wedge, a cement block, a titanium metal wedge and a titanium metal block (see Figs. 1 and 2). Extra bone resection was necessary with the use of block augments. Tibial geometry was obtained through the International Society of Biomechanics Finite Element Repository managed by the Istituti Ortopedici Rizzoli, Bologna, Italy [11]. Stem extensions of $30 \mathrm{~mm}$ were also employed with all models to examine the effect of these on stress patterns. A $1 \mathrm{~mm}$ thick bone cement layer was used to affix all components. The models contained both cortical and cancellous bone regions. The cortical bone was modelled as a $2 \mathrm{~mm}$ thick layer on the lateral and medial sides of the bone.

Plane strain FE analysis was performed using ABAQUS 6.7 (Simulia, Warrington, UK) with a thickness of $27 \mathrm{~mm}$ assigned to the elements for out-of-plane stiffness contribution to the model. Eight-noded quadrilateral reduced integration elements were used in all models. Table 1 shows the material properties for the different materials used in the models $[\mathbf{1 2}, \mathbf{1 3}]$. No relative motion between the various interfaces was permitted and all components were assumed to be isotropic, 
homogenous and linearly elastic. A convergence study was undertaken to ensure a sufficient number of elements were used in the models. The final mesh used for cases with and without stem extensions can be seen in Fig 3. The distal end of the tibia was constrained in all directions. A joint reaction force (JRF) of $2058 \mathrm{~N}$ (3 times body weight of a $70 \mathrm{~kg}$ person) was applied with an even distribution to both tibial bearing condyles. The JRF and loading distribution were considered appropriate for this analysis as research has shown the maximum load to be approximately 3 times body weight during level walking [14] and equivalent geometries of the tibial condyles were assumed. Two main regions of interest (ROIs) within the cancellous bone were identified (see Fig. 2) at which equivalent stresses were considered in detail. ROIs consisted of a $2 \times 2$ block of elements and were chosen as regions which are prone to early failure in TKR. Shear stresses in the cement along the margin of the defect (i.e. the cement-bone interface) were plotted to investigate if these would lead to early failure. Stress values were also examined for all models with $30 \mathrm{~mm}$ stem extensions to assess the effect of the inclusion of a stem on the stress distribution.

\section{RESULTS}

Fig. 3 shows an overall equivalent stress profile of the $15^{\circ}$ metal block augment model and its corresponding stem extension model. It can be seen that the stem transmits more load distally and hence the stem stresses are higher in the stem extension model. Consequently the overall proximal cancellous stresses are lower. However due to the increased rigidity of the implant in the stem extension model (afforded by the closer proximity of the distal part of the stem to the cortical bone) the implant tray is also more highly stressed compared with the model without a stem extension (see Fig. 4). Thus the cancellous bone adjacent to the tray and stem (i.e. 
ROI 1) is likely to experience higher equivalent stress levels in the stem extension model. The value of the central tibial tray displacement in the axial direction (i.e. vertical) was found to be significantly higher in all models without a stem (e.g. 0.09 $\mathrm{mm}$ for block augment $15^{\circ}$ defect model) compared to those with a stem (e.g. 0.06 $\mathrm{mm})$. Again this is due to the increased rigidity afforded by the closer proximity of the distal stem to the cortical bone.

\subsection{Equivalent Stress in the Cancellous bone ROIs}

\subsection{1 $15^{\circ}$ Defect}

The results are presented as mean equivalent stress \pm standard error of the mean at the ROIs. Equivalent stress values were found to be significantly higher $(\mathrm{p}<0.05)$ in the ROI 1 in the model with no stem extension where the cement wedge $(0.34 \pm 0.06$ $\mathrm{MPa})$ was used to fill the defect when compared with the cement block $(0.23 \pm 0.05$ $\mathrm{MPa})$, metal wedge $(0.23 \pm 0.06 \mathrm{MPa})$ and metal block $(0.11 \pm 0.04 \mathrm{MPa})$ components. These results are shown graphically in Fig 5. When a $30 \mathrm{~mm}$ stem extension was employed equivalent stresses were found to be significantly higher than without a stem in all cases; the metal block $(0.27 \pm 0.09 \mathrm{MPa})$, the cement block $(0.46$ $\pm 0.07 \mathrm{MPa})$, the cement wedge $(0.52 \pm 0.09 \mathrm{MPa})$ and the metal wedge $(0.45 \pm 0.1$ $\mathrm{MPa}$ ) models. The ordering of the models according to the stress value was the same as with no extensions; i.e. the cement wedge followed by cement block and metal wedge and finally the metal block.

In ROI 2 similar trends were found with the highest stresses found in the cement wedge $(0.89 \pm 0.03 \mathrm{MPa})$ In this region the stresses with the cement block $(0.89 \pm 0.03 \mathrm{MPa})$ were similarly high compared with the metal block $(0.74 \pm 0.03$ $\mathrm{MPa})$ and metal wedge $(0.74 \pm 0.05 \mathrm{MPa})$ augment models respectively. However, in 
contrast to ROI 1, significantly lower stresses were found in ROI 2 when a $30 \mathrm{~mm}$ stem extension was employed in all cases. Result values are shown graphically in Fig. 6.

\subsection{2 $30^{\circ}$ Defect}

For the $30^{\circ}$ defect the equivalent stress values in ROI 1 and ROI 2 followed broadly similar trends to those found with the $15^{\circ}$ defect. The stresses were significantly lower for the metal block $(0.12 \pm 0.03 \mathrm{MPa})$, followed by the cement block $(0.13 \pm$ $0.03 \mathrm{MPa})$, metal wedge $(0.16 \pm 0.05 \mathrm{MPa})$ and cement wedge $(0.32 \pm 0.06 \mathrm{MPa})$ models. Again, when a $30 \mathrm{~mm}$ stem extension was introduced the stress values were significantly increased in all models.

In ROI 2 equivalent stresses were again highest in the case of the cement wedge $(1.34 \pm 0.17 \mathrm{MPa})$ followed by the metal wedge $(1.19 \pm 0.3 \mathrm{MPa})$, the cement block $(1.09 \pm 0.04 \mathrm{MPa})$ and the metal block $(1.01 \pm 0.08 \mathrm{MPa})$. Significantly lower equivalent stresses were again found in all models when a stem extension of $30 \mathrm{~mm}$ was employed.

\subsection{Shear Stress Analysis}

\subsection{1 $15^{\circ}$ Defect}

The results are presented as a plot of the shear stresses along the margin of the defect $6 \mathrm{~mm}$ from the cortical end to the tibial stem. Shear stress values were found to be significantly lower along the margin of the defect for the metal block when compared with the cement block. Similarly for the wedge shaped augments stress values were significantly lower for the metal wedge when compared with the cement wedge. 
Overall no significant difference was recorded between the metal wedge and metal block components. The results are shown graphically in Fig. 7. Shear stress values were significantly increased in all models when a $30 \mathrm{~mm}$ stem extension was employed except for the metal block component model where results were significantly similar.

\subsection{2 $30^{\circ}$ Defect}

Shear stresses values were again significantly higher for the cement wedge compared with the other models without a stem extension. No significant difference was recorded for shear stress values between the metal wedge, metal block and cement block component models. When a $30 \mathrm{~mm}$ stem extension was introduced the shear stress values were significantly increased in all cases except for the metal block component model where values were significantly similar. Results are shown graphically in Fig. 8.

\section{DISCUSSION}

The objective of this study was to determine if the incidence of early failure of TKR components with moderate uncontained type- 2 defects can be reduced through the use of metal components instead of the use of cement alone. Other objectives include whether a metal block component is more stable than a metal wedge construct consequently reducing the need to use a stem extension. The quantity of bone resection is an important aspect of any joint replacement surgery. Thus the main disadvantage of the use of a block augment is the loss of proximal bone but this may be alleviated by improved stability and reduced stress concentrations. Previous invitro studies [7] have indicated that the use of metal wedge constructs to treat $20^{\circ}$ 
defects can increase the stiffness recorded when compared with cement wedges and thus improve the rigidity of the system. However the increases in stiffness values were slight (i.e. not statistically significant) and thus this may be due to the increased rigidity afforded by the material properties of the metal component. This study aimed to use the FE method to determine if equivalent stress concentrations in the cancellous bone and shear stresses in the cement along the margin of the defect can predict the relative success of different augment types.

Increased equivalent stress concentrations were predicted in the cancellous bone in ROI1 in the cement wedge and cement block augment models when compared with the metal wedge and metal block augment models respectively. It is suggested that these increased stresses increase the possibility of loosening of the cement augments. In the case of both defect angles the metal block augments produced the lowest stress concentrations. This matches clinical theories where it is commonly believed that stress concentrations on the defect margin are responsible for early loosening or fracture of the cement mantle [8]. The stresses with metal augments may be lower than corresponding cement augments because the metal stiffens the tibial tray, reducing the stresses in the cancellous region below. The taper may have higher stresses than the corresponding block as the block transfers the load more distally in the cancellous region. Equivalent stresses were also higher near to the junction of the augment and the cortical bone (i.e. ROI 2) for the cement filled augment models compared with their corresponding metal augment models thus increasing the possibility of loosening at this region. Again this can be attributed to the greater tray stiffening afforded by the metal augments.

Shear stresses were also higher for the use of cement wedge and cement block augments when compared with that of metal wedge and metal block augments 
respectively for the $15^{\circ}$ defect. Thus the sacrifice of native bone in order to create the block profile to treat defected bone may be necessary to increase the longevity of TKR tibial replacements. The authors do not recommend the use of cement wedges or cement blocks to fill large proximal tibial defects of $15^{\circ}$ and $30^{\circ}$. The authors also favour the use of metal block augments over metal wedge augments due to their reduced equivalent stress concentrations in the cancellous ROIs. It is interesting that there is no significant difference between metal block and metal wedges in shear stresses along the margin of the defect. This is in contrast to the accepted belief that shear stresses should be higher on the angled wedge surface when compared with the flat block surface. Closer investigation of the shear stresses show that positive shear in Figs 7 and 8 pull the cancellous bone inwards (i.e. towards the tibial stem). This occurs because the lower modulus cancellous bone displaces more in the mediallateral direction (under the influence of the compressive loading) than the adjacent higher modulus metal tray and augment. Thus the tray and augment act to restrict the lateral expansion of the bone through shear stresses acting toward the centre of the tibia. These shear stresses act in the opposite direct to any shear stresses caused by the inclination of the tapered augment to the direction of loading (which will act on the bone in a laterally outward direction (i.e. away from the tibia centre line). In the 2D model the cortical shell cannot produce hoop stresses and thus will not provide the medial and lateral constraint that exists in the real tibia. Thus this effect may be due to a limitation of the 2D representation. However, this lateral expansion effect will also be present (albeit to a lesser extent) in the actual tibia and full 3D modelling will enable this to be further assessed.

The use of stem extensions was found to increase equivalent stress concentrations at ROI1 in all models. This stress increase is due to the reduction of 
displacement of the extended implant stem, as it is closer to the cortical shell in the extended position as shown in Fig 3. This increased the rigidity of the central implant stem which caused increased bending stresses in the implant tray, (again seen in Fig 3.) which in turn produced higher stresses in the adjacent proximal cancellous bone. This is again in contrast to the commonly accepted belief that the addition of a stem extension provides an additional path for load transfer so lowering the load transfer and hence cancellous bone stresses in the proximal region. In contrast, in ROI2 the use of a stem extension reduced the cancellous bone stresses. The reduction in the equivalent stress concentrations for the $30^{\circ}$ defect was approximately double that of the $15^{\circ}$ defect. In addition the use of a stem extension was shown to increase the cement shear stresses in all cases except the metal block augment for both defect sizes. Based on these results the authors do not recommend the use of a stem extension with a metal block augment for smaller defects but these results will need to be verified by a $3 \mathrm{D}$ analysis.

There are limitations associated with the FE model which are likely to affect the results detailed. These include $2 \mathrm{D}$ plane strain assumptions, the absence of ligament and muscle forces, simplified tibial geometry, idealised material behaviour and the assumption of quasi-static and symmetrical condylar loading. Future work will involve validating the above findings through extension of this FE modeling work to that of 3D models.

\section{CONCLUSION}

The use of block augmentation in the treatment of moderate uncontained type-2 defects in TKR results in increased bone resection compared with that of wedge augmentation. This $2 \mathrm{D}$ analysis shows that using metal rather than cement 
augmentation causes a reduction in stress concentrations in both ROIs in the cancellous bone and in the cement shear stresses along the defect margin. The use of metal block augmentation is shown to further reduce the stress concentrations compared with the use of metal wedge augmentation. It has been shown that the use of a stem extension is more beneficial, in terms of stress reduction in the cancellous bone generally (ROI2), in the case of larger defects. Thus the use of stem extensions may not be necessary in the treatment of smaller defects with metal block augmentation. Future work will involve the use of 3D analysis to verify results.

\section{ACKNOWLEDGEMENTS}

This study was supported by the Engineering and Physical Sciences Research Council (EPSRC) and DePuy International Ltd.

\section{REFERENCES}

1. Engh, G. A. and Ammeen, D. J. Bone loss with revision total knee arthroplasty: defect classification and alternatives for reconstruction. Instr Course Lect, 1999, 48, 167.

2. Radnay, C. S. and Scuderi, G. R. Management of bone loss: augments, cones, offset stems. Clin. Orthop. Rel. Res., 2006, 446, 83-92.

3. Harada, Y., Wevers, H. W. and Cooke, T. D. V. Distribution of bone strength in the proximal tibia. J. Arthroplasty, 1988, 3(2), 167-175.

4. Dennis, D. A. The structural allograft composite in revision total knee arthroplasty. J. Arthroplasty. 2002, 17(4), 90-93.

5. Brooks, P. J., Walker, P. S. and Scott, R. D. Tibial component fixation in deficient tibial bone stock. Clin. Orthop. Relat. Res., 1984, 205, 153-165. 
6. Brand, M. G., Daley, R. J., Ewald, F. C. and Scott, R. D. Tibial tray augmentation with modular metal wedges for tibial bone stock deficiency. Clin. Orthop. Relat. Res., 1989, 248, 71-79.

7. Jeffrey, R. S., Orton, M. A. and Denham, R. A. Wedged tibial components for total knee arthroplasty. J. Arthroplasty, 1994, 9(4), 381-387.

8. Chen, F. and Krackow, K. A. Management of tibial defects in total knee arthroplasty. Clin. Orthop. Rel. Res., 1994, 305, 249-257.

9. Mabry, T. M. and Hanssen, A. D. The role of stems and augments for bone loss in revision knee arthroplasty. J. Arthroplasty, 2007, 22(4), 56-60.

10. Meneghini, M., Lewallen, D. G. and Hanssesn, A. D. Use of porous tantalum metaphyseal cones for severe tibial bone loss during revision total knee replacement. J. Bone Jt Surg. Am., 2008, 90(1), 78-84.

11. ISB, 2007: International Society of Biomechanics website: http://www.isbweb.org/. Website accessed in 2007.

12. Lewis, G., Jonathan, V. and Kambhampati, S. Effect of material property representation on stresses in endoprostheses. Biomed Mater Eng, 1998, 8(1), $11-23$.

13. Nyman, J. S., Hazelwood, S. J., Rodrigo, J. J., Martin, R. B. and Yeh, O. C. Long stemmed total knee arthroplasty with interlocking screws: a computational bone adaptation study. J. Ortho. Res., 2004, 22, 51-57.

14. Morrison, J. B. The mechanics of the knee joint in relation to normal walking. J. Biomechanics, 1970, 3, 51-61. 


\begin{tabular}{ccc}
\hline Material & E (GPa) & v \\
\hline PMMA (Cement) & 2.15 & 0.46 \\
Ti4A16V (Tibial Component) & 110 & 0.36 \\
Cortical Bone & 17 & 0.3 \\
Cancellous Bone & 0.7 & 0.3 \\
UHMWPE (Bearing) & 2.3 & 0.25 \\
\hline
\end{tabular}
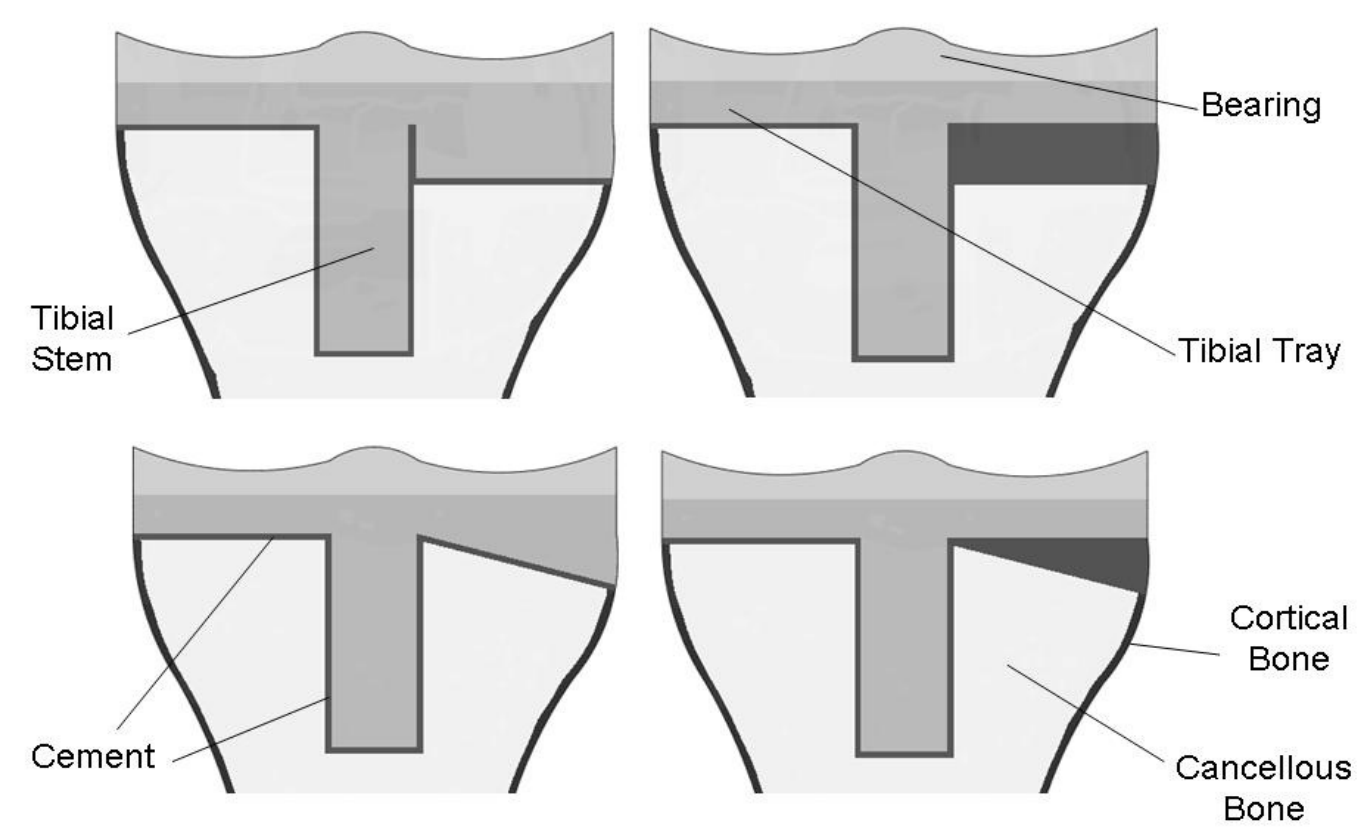

Fig. 1 2D proximal tibia (distal area removed for clarity) of $15^{\circ}$ defect models with (from top left clockwise) metal block, cement block, cement wedge and metal wedge augments. 

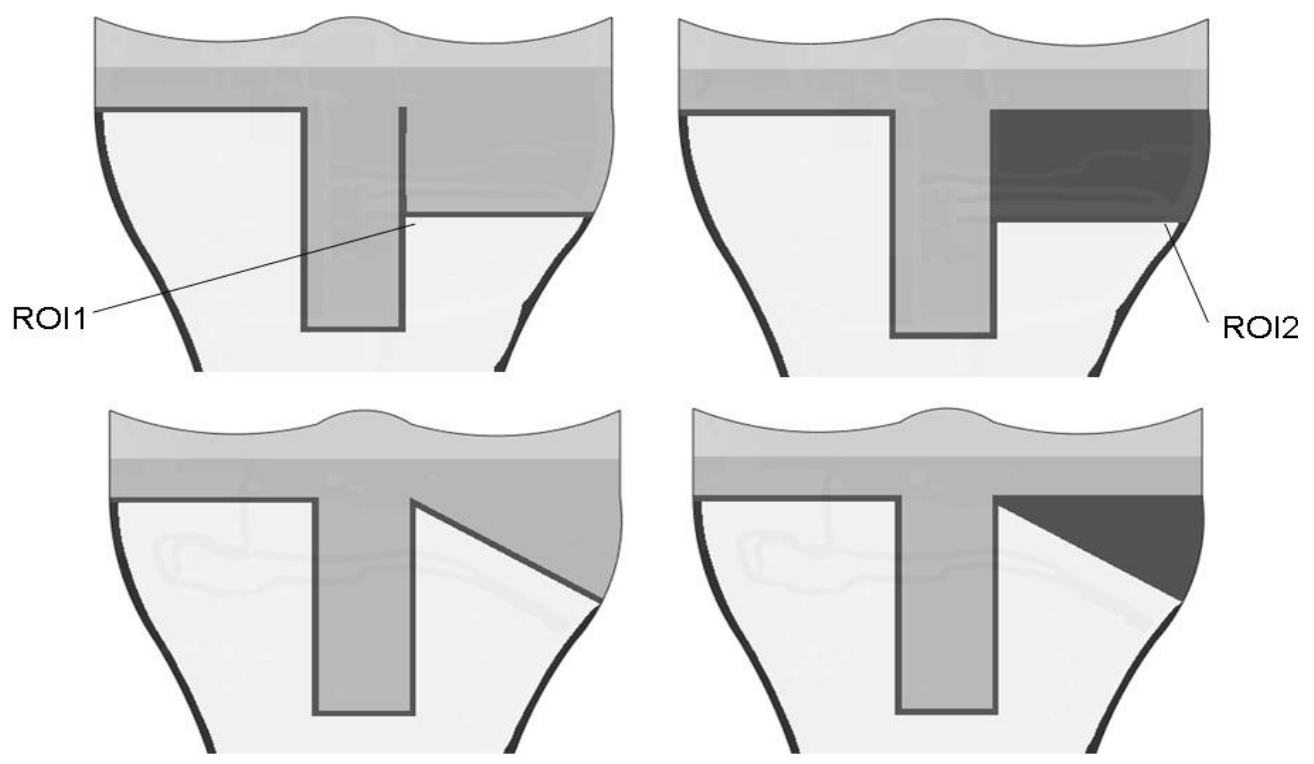

Fig. 2 2D proximal tibia (distal area removed for clarity) showing $30^{\circ}$ defect models with (from top left clockwise) metal block, cement block, cement wedge and metal wedge augments and ROIs investigated.

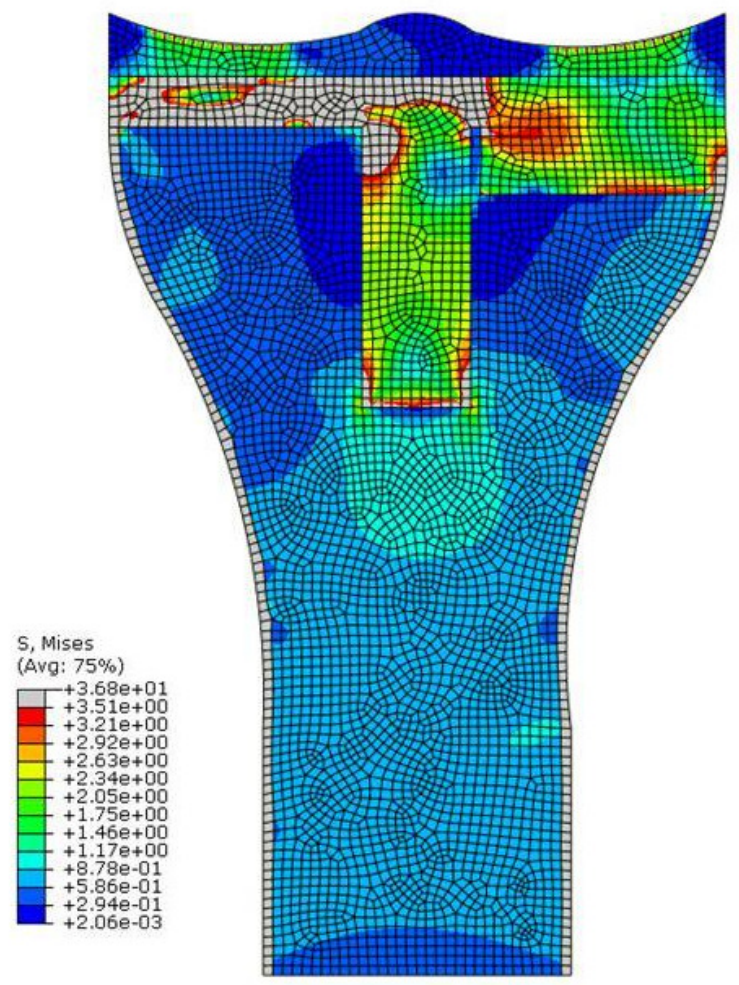

(a)

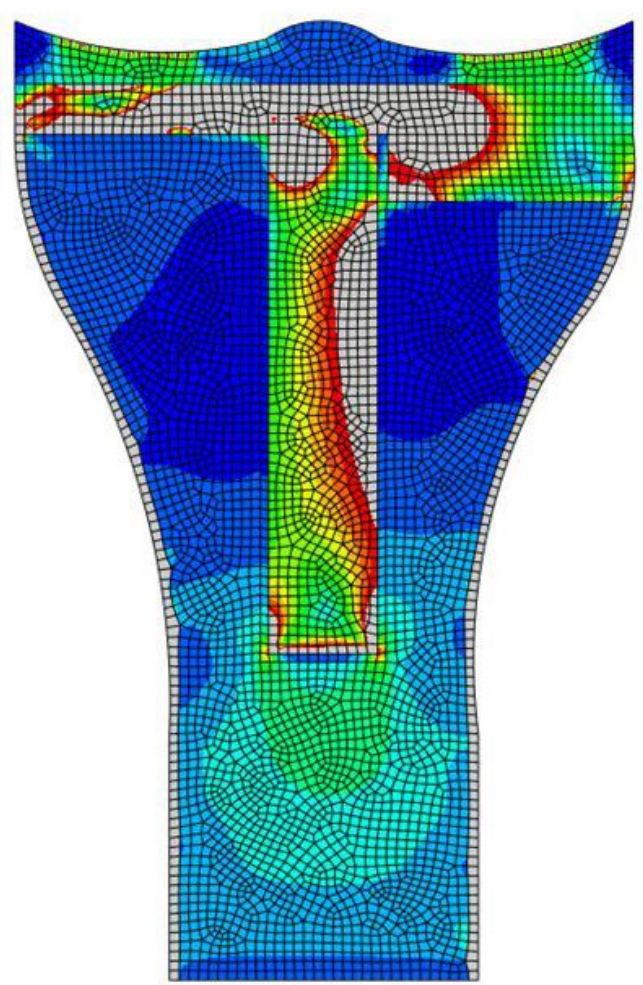

(b) 
Fig. 3 Equivalent stress profiles of the $15^{\circ}$ TKR metal block augment model showing (a) without and (b) with stem extension.

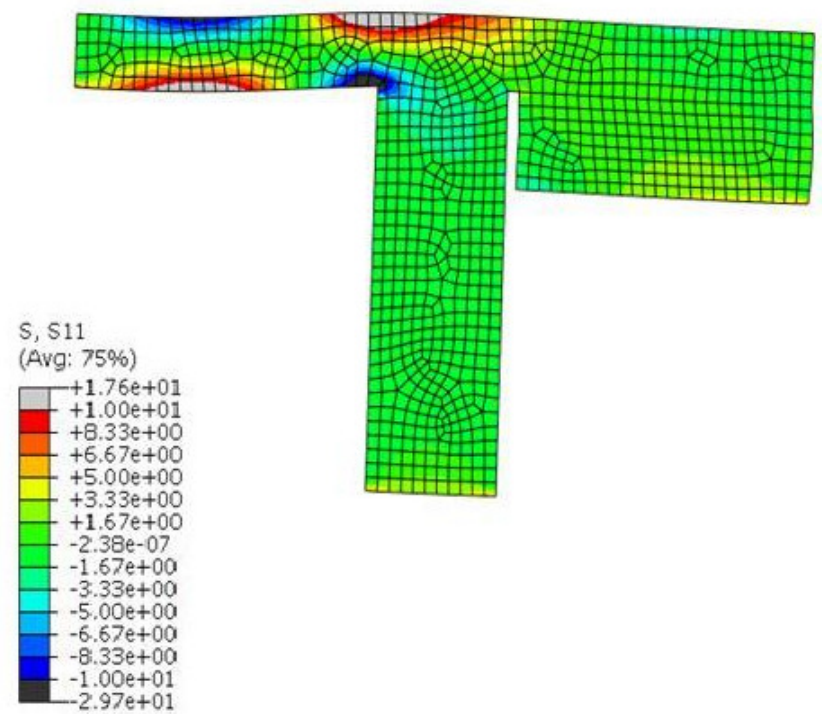

(a)

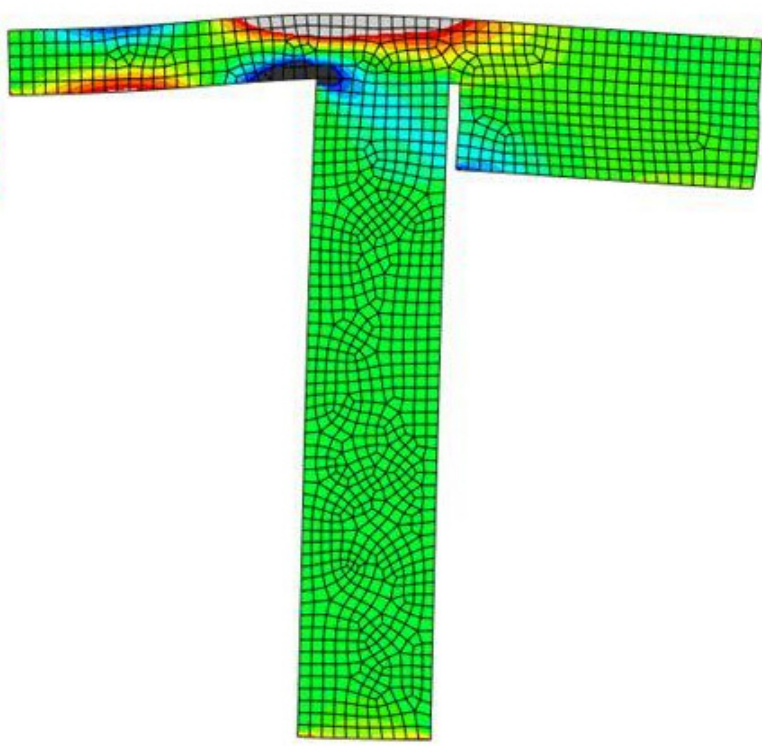

(b)

Fig. 4 Deformed stress profiles in the x-direction of the $15^{\circ}$ TKR tibial tray, stem and metal block augment model showing (a) without and (b) with stem extension.

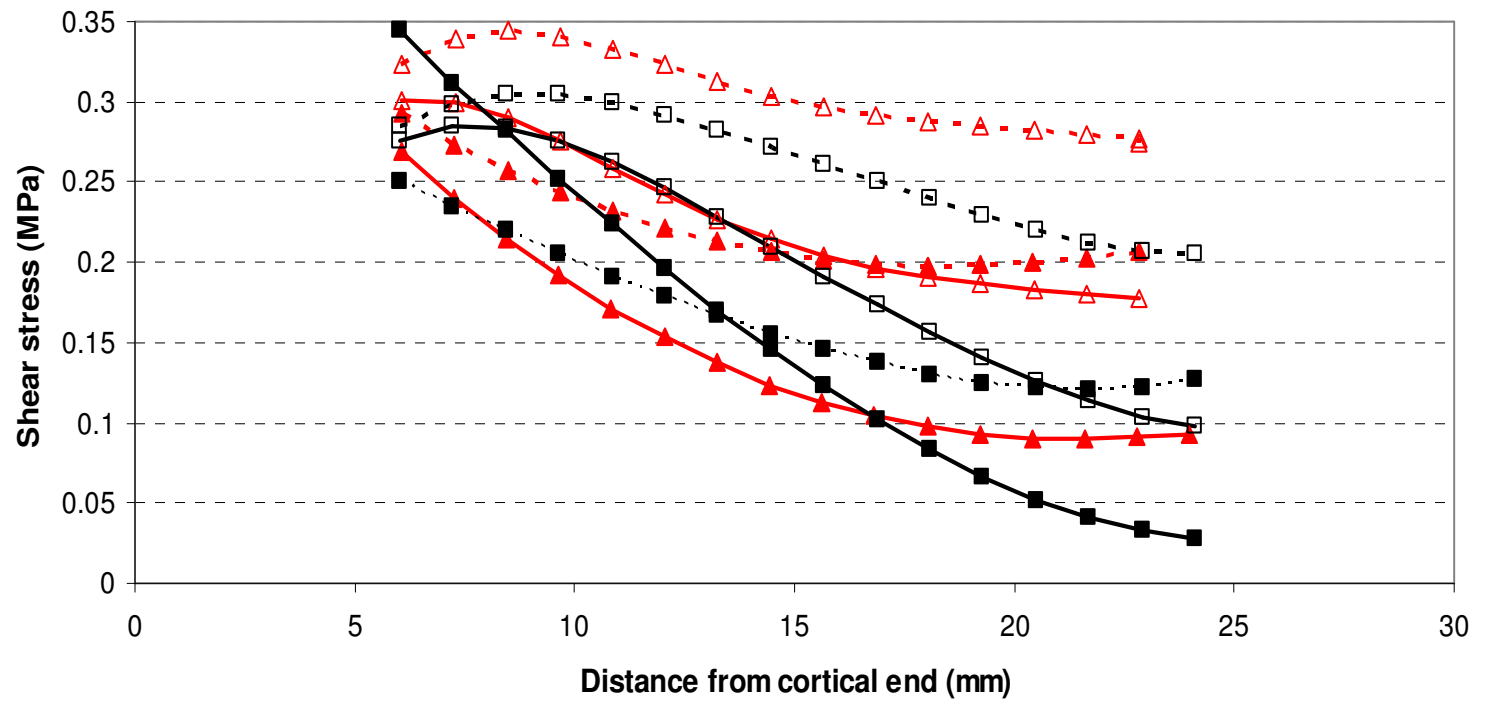

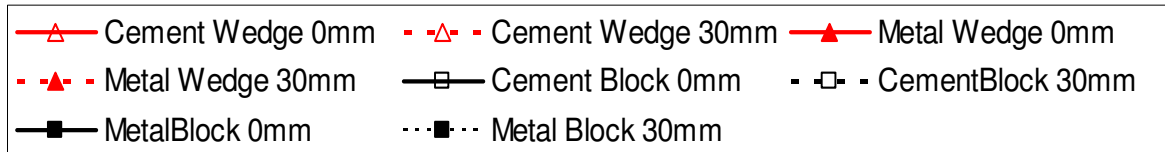


Fig. 7 Average shear stress values of different augment procedures for $15^{\circ}$ defect along the margin of the defect.

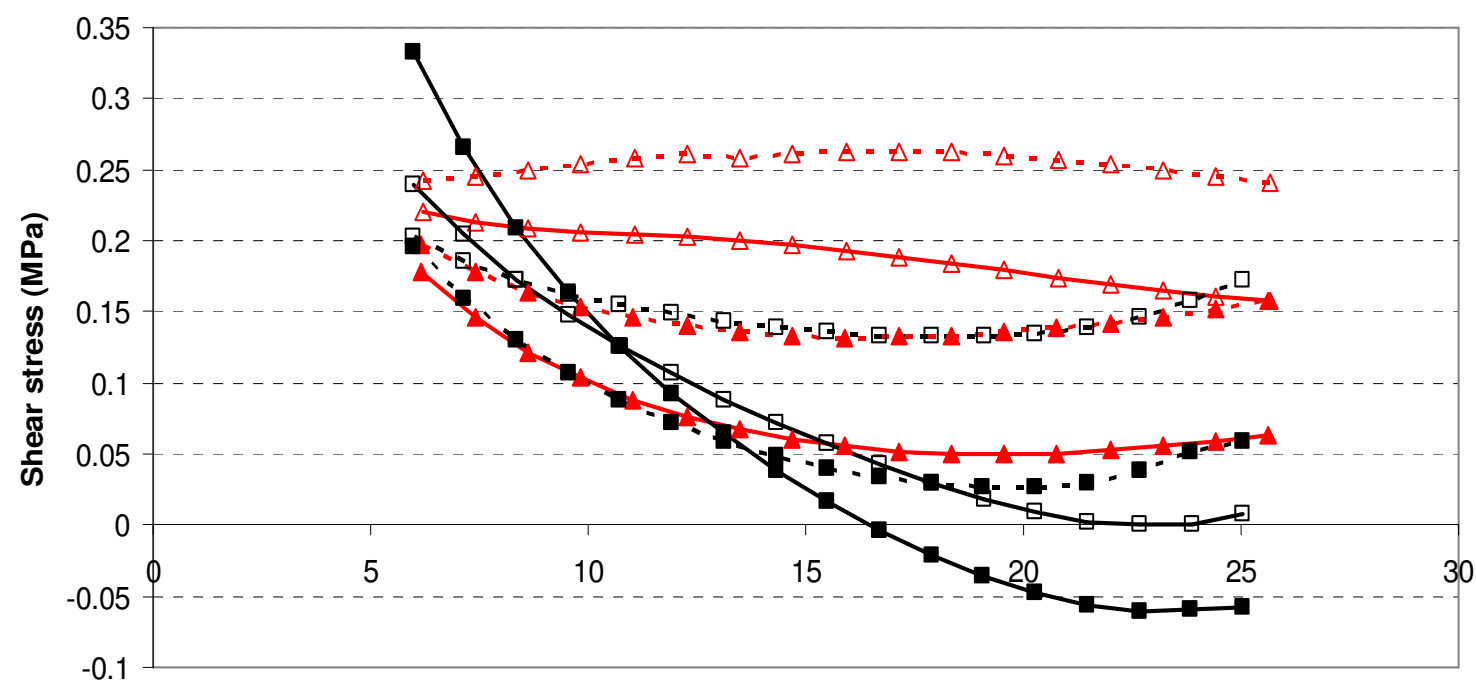

Distance from cortical end (mm)

\begin{tabular}{|c|c|}
\hline $\begin{array}{l}\triangle \triangle \text { Cement Wedge } 0 \mathrm{~mm} \\
-\Delta-\text { - Metal Wedge } 30 \mathrm{~mm} \\
\rightarrow-\text { Metal Block } 0 \mathrm{~mm}\end{array}$ & $\begin{array}{l}-\triangle-\text { - Cement Wedge 30mm } \longrightarrow-\text { Metal Wedge 0mm } \\
\square-\text { Cement Block 0mm } \quad-\square-\text { - Cement block 30mm } \\
-\square-\text { - Metal Block 30mm }\end{array}$ \\
\hline
\end{tabular}

Fig. 8 Average shear stress values of different augment procedures for $30^{\circ}$ defect along the margin of the defect.

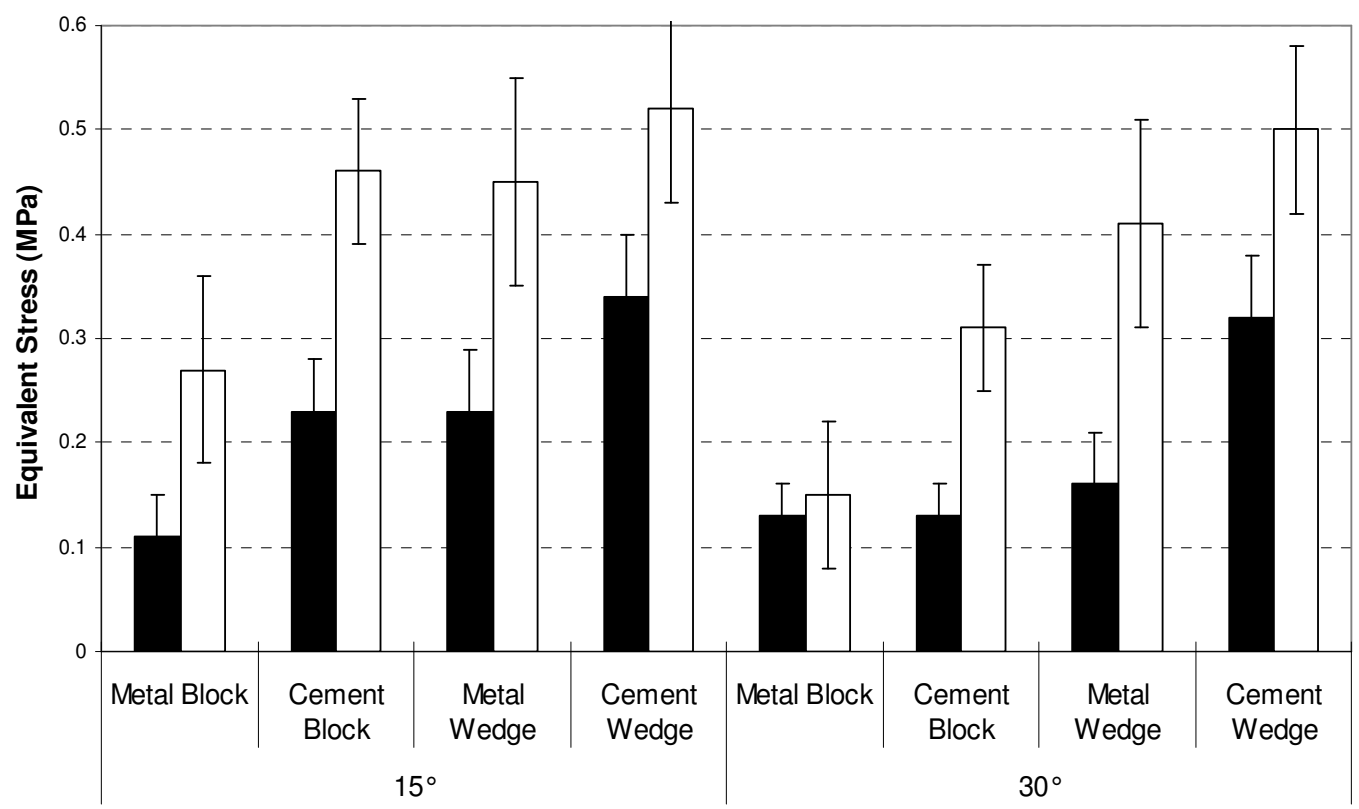

Augment

No Stem Extension $\square$ 30mm Stem Extension 
Fig. 5 Equivalent stress values (mean \pm standard deviation) of different augment procedures for $15^{\circ}$ and $30^{\circ}$ defects for ROI 1 .

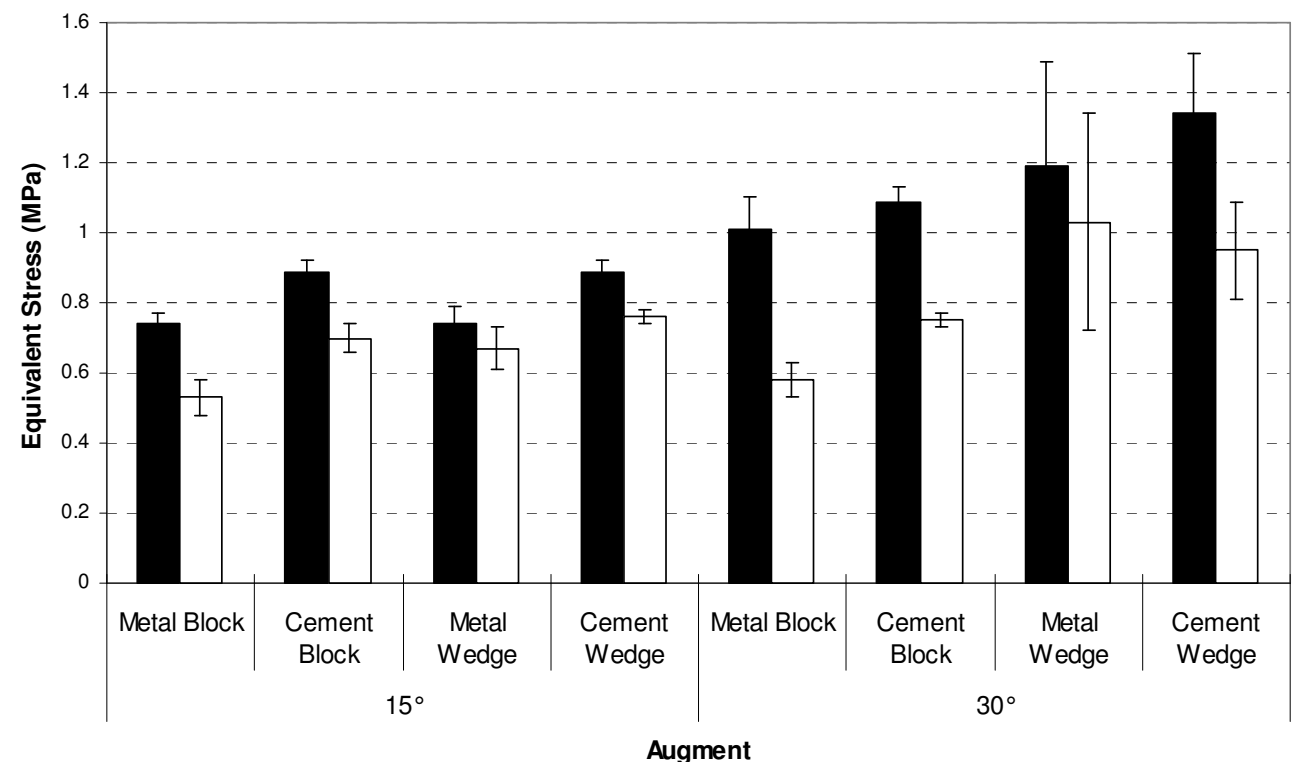

$\square$ No Stem Extension $\square$ 30mm Stem Extension

Fig. 6 Equivalent stress values (mean \pm standard deviation) of different augment procedures for $15^{\circ}$ and $30^{\circ}$ defects for ROI 2. 